Abstract 52 Table 1 CPAP compliance (\% of usage $>4$ hours per night) and average hours of CPAP use per night in both study groups

\begin{tabular}{|c|c|c|c|c|c|}
\hline & & $\begin{array}{l}\text { Number of } \\
\text { patients }\end{array}$ & Median & IQR & P value \\
\hline \multirow[t]{2}{*}{$\begin{array}{l}\text { CPAP compliance } \\
\text { (in \%) }\end{array}$} & $\begin{array}{l}\text { Pre- COVID } \\
\text { group }\end{array}$ & 110 & 38.50 & 69 & \multirow{2}{*}{0.141} \\
\hline & $\begin{array}{l}\text { Post- COVID } \\
\text { group }\end{array}$ & 98 & 57.00 & 85 & \\
\hline \multirow[t]{2}{*}{$\begin{array}{l}\text { Average CPAP } \\
\text { use (in hours) }\end{array}$} & $\begin{array}{l}\text { Pre- COVID } \\
\text { group }\end{array}$ & 110 & 3.02 & 5.06 & \multirow[t]{2}{*}{0.034} \\
\hline & $\begin{array}{l}\text { Post- COVID } \\
\text { group }\end{array}$ & 98 & 4.46 & 5.40 & \\
\hline
\end{tabular}

Abstract 52 Table 2 CPAP compliance ( $\%$ of CPAP usage $>4$ hours/night) across the categories

\begin{tabular}{|c|c|c|c|c|c|c|c|}
\hline & & \multicolumn{3}{|c|}{ Pre- COVID group } & \multicolumn{3}{|c|}{ Post- COVID group } \\
\hline & & Median & IQR & $P$ value & Median & $\mid Q R$ & P value \\
\hline \multirow[t]{2}{*}{ Gender } & Male & \begin{tabular}{|l|}
36.0 \\
\end{tabular} & 61 & \multirow[t]{2}{*}{0.48} & 55.0 & 84 & \multirow[t]{2}{*}{\begin{tabular}{|l|l|}
0.99 \\
\end{tabular}} \\
\hline & Female & \begin{tabular}{|l|}
50.0 \\
\end{tabular} & 77 & & 70.0 & 87 & \\
\hline \multirow[t]{4}{*}{ Ethnicity } & British white & \begin{tabular}{|l|l|}
47.0 \\
\end{tabular} & 70 & \multirow{4}{*}{0.119} & 63.0 & 77 & \multirow{4}{*}{0.347} \\
\hline & BAME & \begin{tabular}{|l|l|}
36.5 \\
\end{tabular} & 69 & & 24.5 & 74 & \\
\hline & Other white & \begin{tabular}{|l|}
25.0 \\
\end{tabular} & 42 & & 80 & 80 & \\
\hline & Not stated & \begin{tabular}{|l|}
10.0 \\
\end{tabular} & 32 & & 25.0 & 93 & \\
\hline \multirow[t]{2}{*}{$\begin{array}{l}\begin{array}{l}\text { Anti- } \\
\text { depressant }\end{array}\end{array}$} & $\begin{array}{l}\text { Not on } \\
\text { antidepressants }\end{array}$ & 32.0 & 63 & \multirow[t]{2}{*}{0.03} & 55.0 & 85 & \multirow[t]{2}{*}{0.94} \\
\hline & $\begin{array}{l}\text { On } \\
\text { antidepressants }\end{array}$ & 69.0 & 71 & & 63.0 & 76 & \\
\hline \multirow{2}{*}{$\begin{array}{l}\text { Daytime } \\
\text { sleepiness }\end{array}$} & ESS $\leq 10$ & 27.5 & 51 & \multirow{2}{*}{0.049} & 63.0 & 81 & \multirow{2}{*}{0.722} \\
\hline & ESS $>10$ & 51.0 & 74 & & \begin{tabular}{|l|}
42.0 \\
\end{tabular} & 90 & \\
\hline \multirow{3}{*}{$\begin{array}{l}\text { OSA } \\
\text { severity }\end{array}$} & Mild OSA & 22.0 & 50 & \multirow{3}{*}{0.08} & 78.0 & 97 & \multirow{3}{*}{0.694} \\
\hline & Moderate OSA & \begin{tabular}{|l|}
27.0 \\
\end{tabular} & 63 & & 57.0 & 92 & \\
\hline & Severe OSA & 55.5 & 76 & & 51.0 & 71 & \\
\hline
\end{tabular}

patients, British white ethnicity and those with moderate and severe OSA, but this did not reach any statistical significance. Compliance was significantly high in patients with high ESS and those on an antidepressant in the Pre-COVID group $(\mathrm{p}=0.049$ and 0.03 , respectively). Twelve patients returned CPAP among pre-COVID $(n=110)$ compared to 14 of postCOVID $(n=98)(p=0.531)$ (table 2).

Conclusion The study showed CPAP compliance of telephonic clinic consultation was slightly better compared to conventional clinic consultation. This new virtual clinic model can be adopted successfully during the challenging COVID times.

\section{PSEUDO-OBSTRUCTIVE EVENTS IN SPINAL MUSCULAR ATROPHY AS A POTENTIAL MARKER FOR DISEASE PROGRESSION}

${ }^{1}$ Sakina Dastagir*, ${ }^{1}$ Hui-Leng Tan, ${ }^{1,2,3}$ Andrew Bush, ${ }^{1,4}$ Federica Trucco. ${ }^{1}$ Royal Brompton Hospital, London, UK; ${ }^{2}$ National Heart and Lung Institute, London, UK; ${ }^{3}$ mmperial Centre for Paediatrics and Child Health, UK; ${ }^{4}$ Dept Paediatric Neuroscience, Guy's and St Thomas NHS trust and Department Paediatric Respiratory Medicine, London, UK

\subsection{6/bmjresp-2021-bssconf.47}

Introduction Sleep disordered breathing (SDB) is common in children with spinal muscular atrophy (SMA) as a result of respiratory muscle weakness. However, SDB events are currently scored according to criteria created for healthy children.

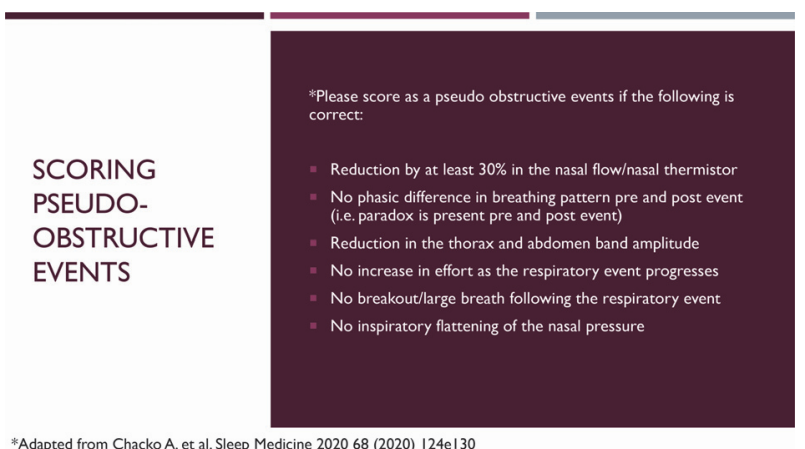

*Adapted from Chacko A et al Sleep Medicine 202068 (2020) |24el30

\section{Abstract 53 Figure 1}

This study aims to add to previous evidence ${ }^{1,2}$ that SMA type II patients have respiratory events (we defined them as 'pseudo-obstruction') which do not conform to the current AASM guidelines for obstructive or central events. They are the result of paradoxical breathing and REM-related shallow breathing.

Methods Respiratory events were defined as either 'obstructive apnoea' (OA), 'central apnoea' (CA), 'central hypopnoea' $(\mathrm{CH})$, 'obstructive hypopnoea' $(\mathrm{OH})$ as per AASM guidelines. We additionally defined the criteria for 'pseudo-obstruction' (PO) based on previous publications (figure 1). ${ }^{1}$

Trained sleep physiologists were provided 8 'test' epochs randomly chosen from either SMA II or other patients. Physiologists were asked to designate the respiratory events they deemed most appropriate for each epoch, blind to diagnosis of the patient. Interscorer reliability tests were performed against the gold standard for each event.

Results The average concordance with the gold standard was $75 \%$ overall. It was mildly reduced to $67 \%$ when looking specifically at POs.

We are currently evaluating whether disease progression is associated with an increase in POs by looking at subsequent yearly sleep studies of 10 SMA II and 1 SMA I patient, selfventilating in room air, across a 3-year period.

Discussion Future efforts will aim to look more closely at inter scorer reliability. Recognising these pseudo-obstructive events may influence treatment. ${ }^{2}$ Additionally, if these events correlate along the motor and respiratory deterioration, they can be used as markers of response to overnight ventilation and, more importantly, to new available treatments.

\section{REFERENCES}

1. Chacko A. Sleep Medicine 2020.

2. Kouri I. Journal of clinical neuromuscular disease, 2020.

3. Berry. AASM manual 2020.

\section{SLEEP SPINDLES AS A BIOMARKER FOR ALPHA- SYNUCLEINOPATHIES IN RAPID EYE MOVEMENT (REM) BEHAVIOUR DISORDER (RBD)}

Eilidh McMillan*, Stevie Williams, Renata Riha. University of Edinburgh, Edinburgh, UK

\subsection{6/bmjresp-2021-bssconf.48}

Introduction Idiopathic rapid eye movement behaviour disorder (iRBD) is a strong predictor for the development of alpha-synucleinopathies. Electroencephalographic (EEG) oscillations known as sleep spindles are found during non-rapid eye movement sleep. These bursts of neural oscillatory activity are 